\title{
Reduced expression of TRF1 is associated with tumor progression and poor prognosis in oral squamous cell carcinoma
}

\author{
HUI-CHING CHUANG ${ }^{1,4^{*}}$, CHANG-HAN CHEN ${ }^{1,4,5^{*}}$, CHAO-CHENG HUANG ${ }^{2,4}$, \\ FU-MIN FANG ${ }^{3,4}$, HSIN-TING TSAI ${ }^{1,4}$ and CHIH-YEN CHIEN ${ }^{1,4}$
}

\begin{abstract}
Departments of ${ }^{1}$ Otolaryngology, ${ }^{2}$ Pathology and ${ }^{3}$ Radiation Oncology; ${ }^{4}$ Kaohsiung Chang Gung Head and Neck Oncology Group, Cancer Center; and ${ }^{5}$ Center for Translational Research in Biomedical Sciences, Chang Gung Memorial Hospital-Kaohsiung Medical Center, Chang Gung University College of Medicine, Kaohsiung, Taiwan, R.O.C.
\end{abstract}

Received September 10, 2010; Accepted November 19, 2010

DOI: $10.3892 / \mathrm{etm} .2010 .168$

\begin{abstract}
The functions of telomeric repeat-binding factor 1 (TRF1) and 2 (TRF2) in oral carcinogenesis are largely unexplored. This study examined the relationship between the expression of TRF1 and TRF2 and clinicopathological variables and survival in oral cavity squamous cell carcinoma (OCSCC). Western blotting and immunohistochemistry were used to evaluate the protein expression of TRF1 and TRF2 in paired OCSCC patient specimens. Expression of TRF1 and TRF2 was assessed by immunohistochemistry in 256 OCSCC patients who underwent tumor resection without previous radiotherapy. The results were analyzed using Fisher's exact test. Protein expression of TRF1 and TRF2 was significantly lower in the OCSCC than in the adjacent non-tumor tissue. Reduced TRF1 and TRF2 levels in 256 patients, as revealed by immunohistochemistry, were significantly associated with aggressive clinicopathological features, such as advanced tumor stage $(\mathrm{p}<0.001)$ and advanced tumor node metastasis stage $(\mathrm{p}<0.001)$. According to Kaplan-Meier analysis, reduced TRF1 expression was significantly correlated with an unfavorable cumulative 5-year overall survival rate $(\mathrm{p}<0.001)$. In conclusion, decreased expression of TRF1 was significantly associated with tumor progression and poor prognosis in OCSCC patients.
\end{abstract}

\section{Introduction}

Oral cavity squamous cell carcinoma (OCSCC) accounts for at least $90 \%$ of all oral malignancies. It is a multifactorial

Correspondence to: $\mathrm{Dr}$ Chih-Yen Chien, Department of Otolaryngology, Chang Gung Memorial Hospital-Kaohsiung Medical Center, Chang Gung University College of Medicine, 123 Ta-Pei Road, Niao Sung Hsiang, Kaohsiung 833, Taiwan, R.O.C.

E-mail: cychien3965@adm.cgmh.org.tw

*Contributed equally

Key words: telomeric repeat-binding factor 1, telomeric repeatbinding factor 2 , oral cavity squamous cell carcinoma condition with etiological links to a wide variety of external causes of cancer, including alcohol, tobacco and betel nut use, and certain viral infections. The high and increasing prevalence of OCSCC in Taiwan has been attributed to the popularity of betel nut chewing. It was estimated that, in 2006, more than 4,000 people in Taiwan were diagnosed with oral cancer. This represents $5.49 \%$ of all newly diagnosed malignancies. Despite advances in technology and the implementation of multidisciplinary treatment programs, only modest improvements in survival rates have been achieved, and these are primarily due to earlier diagnosis, rather than improved therapeutic interventions (1). Moreover, the rate of recurrence of advanced tumors remains relatively high. Salvage outcomes are unsatisfactory, although they depend on the stage of the recurrent tumors (2). Investigation of OCSCC progression from a genetic perspective has identified distinct patterns and timings of genetic alterations (3). The most important prognostic factors in OCSCC are those that form part of the grading system, including tumor stage and lymph node status (4-6). The identification of new prognostic factors linked to OCSCC initiation and progression may aid in the development of new diagnostic tools and treatment strategies.

Among the various molecular factors implicated in carcinogenesis, telomere dysfunction has emerged as an early event associated with genetic instability. Telomeres stabilize the ends of chromosomes, protect them from end-to-end fusion and mediate chromosome pairing during cell division (7-10). Recently, telomere-associated proteins, such as telomeric repeat-binding factor 1 (TRF1) and 2 (TRF2), have been identified as putative modulators of telomerase activity and have been suggested to play key roles in the maintenance of the telomere function $(8,9,11,12)$. Several reports have indicated that the altered expression of TRF1 and TRF2 proteins is associated with tumor progression in various human carcinomas, including lung, stomach, adrenal and pancreatic cancer; the altered expression has also been identified in malignant hematopoietic cells and colorectal pre-neoplastic lesions (13-20). However, the relationship between TRF1 and TRF2 and OCSCC remains unclear. The aim of the present study was to examine TRF1 and TRF2 expression in OCSCC and to determine its relationship with clinicopathological variables and survival. 


\section{Materials and methods}

Patients and tumor samples. The study population included 256 OCSCC patients who underwent primary surgical resection without previous radiotherapy and/or chemotherapy between October 1996 and August 2005. Clinicopathological information for each subject, including gender, age, tumor (T) stage, nodal (N) status, tumor node metastasis (TNM) stage and overall survival, was obtained retrospectively from clinical records and pathological reports. TNM status was classified according to the 1997 American Joint Committee on Cancer (AJCC) system. The study was approved by the Medical Ethics and Human Clinical Trial Committee at Chang Gung Memorial Hospital, Taipei, Taiwan. The patient group comprised 17 women and 239 men, with an average age of 50.9 years (range, 26-87 years). Thirty-nine patients were diagnosed with T1 tumors, 55 with T2, 64 with T3 and 98 with T4. A total of 153 patients had an N status of N0, 38 had N1, 48 had N2b, 13 had N2c and 4 had N3. Thirty-four patients had stage I tumors, 38 stage II, 61 stage III and 123 stage IV.

Immunoblot analysis. For tissue protein extraction, frozen samples (adjacent non-tumor and tumor tissues) were homogenized in RIPA lysis buffer (50 mM Tris-HCl, pH 7.5, $150 \mathrm{mM}$ $\mathrm{NaCl}, 1 \% \mathrm{NP}-40,0.5 \%$ Na-deoxycholate and $0.1 \%$ SDS), and the protein concentrations were quantified using a Bio-Rad Protein assay (Bio-Rad, Hercules, CA, USA). Immunoblotting was performed according to standard procedures. Anti-TRF1 and -TRF2 polyclonal antibodies and the anti-GAPDH monoclonal antibody (Santa Cruz Biotechnology Santa Cruz, CA, USA) were used. The bound primary antibody was detected by incubation with HRP-conjugated secondary antibody (Bio/ Can Scientific, Mississauga, ON, Canada). Blots were developed using the Western Lighting reagent, and protein bands were visualized using X-ray film.

Immunohistochemical analysis. OCSCC and adjacent noncancerous tissue samples were identified by a pathologist based on diagnosis and microscopic morphology. Tumor tissues were fixed in $10 \%$ buffered formalin, embedded in paraffin and decalcified in $10 \%$ EDTA. Formalin-fixed paraffin-embedded tissue was sectioned to a thickness of $4 \mu \mathrm{m}$, and the sections were deparaffinized in xylene and rehydrated in a graded series of ethanol (100, 90, 80 and 70\%). The sections were washed in phosphate-buffered saline (PBS) and treated with $3 \% \mathrm{H}_{2} \mathrm{O}_{2}$ for $30 \mathrm{~min}$ to block the endogenous peroxidase activity. Antigens were retrieved by microwaving the sections in $10 \mathrm{mM}$ citrate buffer, $\mathrm{pH}$ 6.0. The sections were then incubated with anti-TRF1 and -TRF2 antibodies (diluted 1:100) for $1 \mathrm{~h}$, washed in PBS and incubated for 30 min with a horseradish peroxidase-Fab polymer conjugate (PicTure ${ }^{\mathrm{TM}}$-Plus kit; Zymed, South San Francisco, CA, USA). After the sections were washed in PBS, the immunoreactive bands were visualized by incubation with 3,3'-diaminobenzidine for $5 \mathrm{~min}$. As a negative control, the primary antibody was omitted. Two pathologists blinded to the subjects' clinical information independently evaluated the reactivity level of the immunostained tissues in 15-20 high-power fields. Criteria were developed for quantitating the immunoreactivity of the TRF1 and TRF2

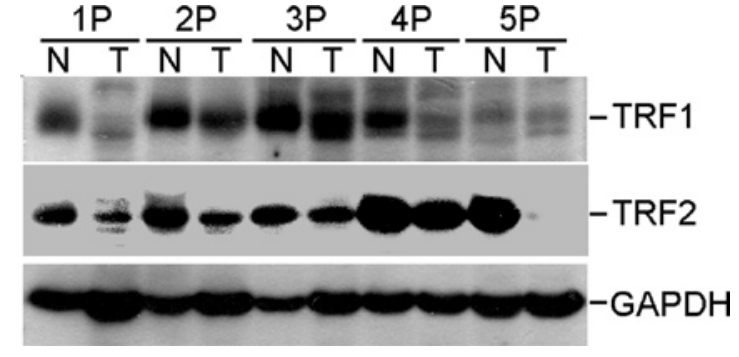

Figure 1. Expression of TRF1 and TRF2 was down-regulated in OCSCC Western blot analysis of the protein expression of TRF1 and TRF2 in paired specimens from 5 oral cancer patients. The total protein extracted from cancerous $(\mathrm{T})$ and adjacent non-cancerous tissues $(\mathrm{N})$ was probed using polyclonal antibodies against TRF1 and TRF2. GAPDH was used as a loading control.

staining in the adjacent non-tumor and tumor sections using a score range of 0 to +3 . A percentage value of 0 indicated $0-25 \%$ of the area stained; $+1,25-50 \% ;+2,50-75 \%$ and +3 , $>75 \%$ stained. Similarly, the staining intensity was graded as $+0,+1,+2$ or +3 . High expression of TRF1 and TRF2 was defined as $\geq+2$ in both scoring methods. Low expression of TRF1 and TRF2 was defined as $\leq+1$ in both scoring methods.

Statistical analysis. Fisher's exact test was used to evaluate the correlation between TRF1 and TRF2 expression and various clinicopathological variables, including gender, age, $\mathrm{N}$ status, $\mathrm{T}$ stage and TNM stage. A p-value $<0.05$ was considered to indicate statistical significance. TRF1 and TRF2 expression and the clinicopathological variables were used in a KaplanMeier analysis of survival, and statistical significance $(\mathrm{p}<0.05)$ was assessed by the log-rank test. To determine the effects of specific prognostic factors on survival, a multivariate analysis was performed using Cox's regression model.

\section{Results}

Down-regulation of TRF1 and TRF2 expression in OCSCC tissues. To investigate the potential roles of TRF1 and TRF2 in the pathogenesis of OCSCC, their expression was assessed in representative and paired tumor and adjacent non-cancerous tissue samples by Western blot analysis using anti-human TRF1 and TRF2 polyclonal antibodies. The protein expression levels of TRF1 and TRF2 were lower in the tumor samples compared to those in the paired non-cancerous tissues (Fig. 1).

TRF1 and TRF2 expression in the tumor and adjacent non-cancerous tissues from the 256 OCSCC patients was also examined immunohistochemically. Representative results of TRF1 and TRF2 immunostaining are presented in Fig. 2A. Staining was stronger in the adjacent non-cancerous tissues (Fig. 2A-a and -f) than the tumor tissues (Fig. 2A-b and -c, TRF1; Fig. 2A-g and -h, TRF2). Moreover, TRF1 and TRF2 expression levels in the tumor samples were negatively correlated with T stage (Fig. 2A-b and -c, TRF1; Fig. 2A-g and -h, TRF2) and N stage (Fig. 2A-d and -e, TRF1; Fig. 2A-i and -j, TRF2). Notably, TRF1 and TRF2 were focally expressed in the nuclei of both tumor and non-cancerous cells (Fig. 2A-b and -c, TRF1; Fig. 2A-g and -h, TRF2). 
A

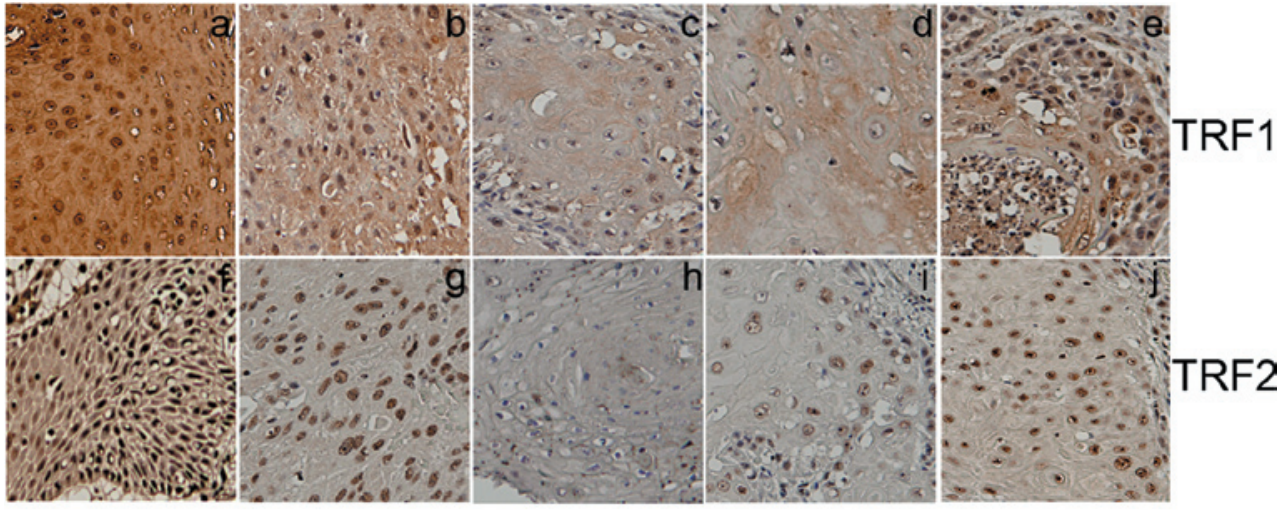

B
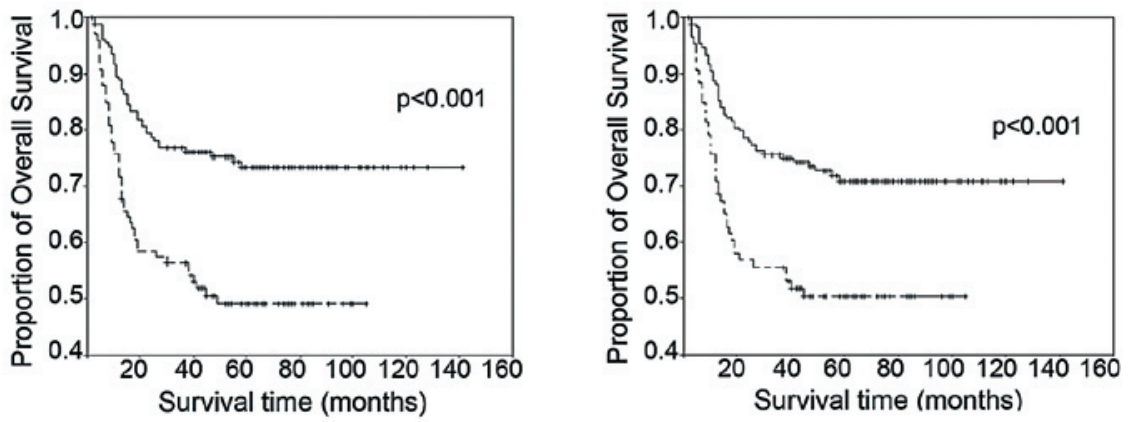

$\overline{\text { TRF2 }}$ high expression

TRF2 low expression

Figure 2. Immunohistochemical staining of TRF1 and TRF2 and overall survival in OCSCC patients. (A) Immunohistochemical staining intensities of TRF1 and TRF2 in tumor and non-cancerous tissues. Non-cancerous tissues displayed extremely strong TRF1 and TRF2 staining in the nucleus (a and f). Staining intensity for TRF1 and TRF2 was lower in the tumor tissues (b,c and g,h). Significant nuclear expression of TRF1 and TRF2 was observed in early (b and $\mathrm{g}$ ) and late (c and h) stage tumors. TRF1 and TRF2 expression was present in patients with (d and i) and without (e and j) lymph node metastases. Original magnification, x100. (B) The survival time of patients with low (-/+) expression of TRF1 and TRF2 was significantly shorter (p<0.001, log-rank test) than that of patients displaying higher $(++/+++)$ expression levels $(n=256)$.

To investigate whether the expression of TRF1 and TRF2 is associated with various prognostic factors, including age, gender and TNM pathologic classification, we classified the patients into two groups based on the immunohistochemical analysis: low (-/+) and high (++/+++) TRF1 or TRF2 expression. Low TRF1 expression was correlated with advanced $\mathrm{T}$ stage $(\mathrm{p}<0.001)$ and advanced TNM stage $(\mathrm{p}<0.001)$. Low TRF2 expression was correlated with advanced $\mathrm{T}$ stage $(\mathrm{p}<0.001)$ and advanced TNM stage $(\mathrm{p}<0.001)$, as well as positive lymph node metastasis $(\mathrm{p}=0.022)$. Neither TRF1 nor TRF2 expression was correlated with age or gender (Table I). These findings suggest that TRF1 and TRF2 expression levels may be linked to tumor progression in OCSCC.

TRF1 and TRF2 expression and OCSCC patient survival. In view of the finding that TRF1 and TRF2 expression levels were associated with $\mathrm{T}$ stage, we investigated whether TRF1 and TRF2 expression was correlated with patient prognosis. As shown in Fig. 2B, Kaplan-Meier overall survival analysis revealed that the prognosis of patients with low (-/+) tumor expression of TRF1 and TRF2 was significantly poorer than that of patients displaying higher $(++/+++)$ expression $(\mathrm{p}<0.001)$. Univariate analysis revealed that advanced $\mathrm{T}$ stage $(\mathrm{p}<0.001)$, positive $\mathrm{N}$ stage $(\mathrm{p}<0.001)$, advanced TNM stage $(\mathrm{p}<0.001)$, low TRF1 expression and low TRF2 expression each predicted a significantly worse prognosis for OCSCC patients (Table II). The prognosis was not associated with age or gender. Cox regression analysis revealed that $\mathrm{T}$ stage (95\% CI, 1.585-5.037; RR=2.826; $\mathrm{p}<0.001)$, N status $(95 \% \mathrm{CI}$, 1.966-4.681; RR=3.034; $\mathrm{p}<0.001)$ and TRF1 expression $(95 \%$ CI, 0.391-0.924; $\mathrm{RR}=0.601 ; \mathrm{p}=0.02$ ) were independent prognostic factors for survival. These results clearly indicate that the clinical prognosis for OCSCC patients is affected by the tumor expression of TRF1 and TRF2, and suggest that TRF1 and TRF2 may be good prognostic indicators in OCSCC.

\section{Discussion}

To our knowledge, this is the first investigation of TRF1 and TRF2 expression in primary OCSCC specimens from a large cohort of patients. Our results indicate that the reduced expression of TRF1 and TRF2 is associated with increased tumor aggressiveness and poor prognosis in OCSCC patients. Low expression of TRF1 and TRF2 and advanced T stage and $\mathrm{N}$ stage were correlated with a poor prognosis. As it is difficult to determine a prognosis for these patients, TRF1 and TRF2 staining of oral cancer cells may be helpful in selecting an appropriate therapeutic strategy following surgery. Our findings suggest that TRF1 is a good prognostic indicator in OCSCC and a candidate molecular target for oral cancer therapy. 
Table I. Correlation between the clinicopathological features and expression of TRF1 and TRF2 in the oral squamous cell carcinoma cases.

\begin{tabular}{|c|c|c|c|c|c|c|c|}
\hline \multirow[b]{2}{*}{ Variables } & \multirow{2}{*}{$\begin{array}{c}\text { No. of } \\
\text { patients }\end{array}$} & \multicolumn{2}{|c|}{ TRF1 expression } & \multirow[b]{2}{*}{ P-value } & \multicolumn{2}{|c|}{ TRF2 expression } & \multirow[b]{2}{*}{ P-value } \\
\hline & & Low & High & & Low & High & \\
\hline Gender & & & & 0.452 & & & 0.434 \\
\hline Male & 239 & 95 & 144 & & 83 & 156 & \\
\hline Female & 17 & 5 & 12 & & 4 & 13 & \\
\hline Age (years) & & & & 0.272 & & & 0.333 \\
\hline$<60$ & 202 & 75 & 127 & & 72 & 130 & \\
\hline$\geq 60$ & 54 & 25 & 29 & & 15 & 39 & \\
\hline Tumor stage & & & & $<0.001^{\mathrm{a}}$ & & & $<0.001^{\mathrm{a}}$ \\
\hline $\mathrm{T} 1$ and $\mathrm{T} 2$ & 94 & 15 & 79 & & 11 & 83 & \\
\hline $\mathrm{T} 3$ and T4 & 162 & 85 & 77 & & 76 & 86 & \\
\hline Nodal stage & & & & 0.090 & & & $0.022^{\mathrm{a}}$ \\
\hline Negative & 153 & 53 & 100 & & 43 & 110 & \\
\hline Positive & 103 & 47 & 56 & & 44 & 59 & \\
\hline TNM stage & & & & $<0.001^{\mathrm{a}}$ & & & $<0.001^{\mathrm{a}}$ \\
\hline I and II & 66 & 5 & 61 & & 1 & 65 & \\
\hline III and IV & 190 & 95 & 95 & & 86 & 104 & \\
\hline
\end{tabular}

aStatistically significant

Table II. Cumulative 5-year overall survival rate according to clinicopathological features.

\begin{tabular}{|c|c|c|c|}
\hline Variables & $\begin{array}{l}\text { No. of } \\
\text { patients }\end{array}$ & $\begin{array}{c}\text { Cumulative } \\
5 \text {-year overall } \\
\text { survival rate }(\%)\end{array}$ & P-value \\
\hline Gender & & & 0.1360 \\
\hline Male & 239 & 62.5 & \\
\hline Female & 17 & 81.9 & \\
\hline TRF1 expression & & & $<0.0010^{\mathrm{a}}$ \\
\hline Low & 100 & 73.3 & \\
\hline High & 156 & 49.1 & \\
\hline TRF2 expression & & & $0.0003^{\mathrm{a}}$ \\
\hline Low & 87 & 70.1 & \\
\hline High & 169 & 50.3 & \\
\hline Age (years) & & & 0.1300 \\
\hline$<60$ & 202 & 66.2 & \\
\hline$\geq 60$ & 54 & 55.1 & \\
\hline Tumor stage & & & $<0.0010^{\mathrm{a}}$ \\
\hline $\mathrm{T} 1$ and $\mathrm{T} 2$ & 94 & 84.0 & \\
\hline $\mathrm{T} 3$ and $\mathrm{T} 4$ & 162 & 56.5 & \\
\hline Nodal stage & & & $<0.0010^{\mathrm{a}}$ \\
\hline Negative & 153 & 77.0 & \\
\hline Positive & 103 & 44.7 & \\
\hline TNM stage & & & $<0.0010^{\mathrm{a}}$ \\
\hline I and II & 66 & 92.4 & \\
\hline III and IV & 190 & 53.9 & \\
\hline
\end{tabular}

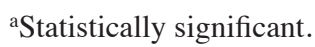

Semi-quantitative RT-PCR analysis of TRF1 and TRF2 mRNA expression in total RNA from tumor samples and matched adjacent non-cancerous tissues from 5 OCSCC patients demonstrated that TRF1 and TRF2 mRNA levels did not differ significantly between the tumor and adjacent non-cancerous tissues (data not shown). This observation suggests that altered TRF1 and TRF2 protein expression during the development of OCSCC may be realized posttranscriptionally.

Telomere-binding proteins have attracted increasing interest due to their essential roles in regulating the length of telomeric DNA tracts and protecting against chromosomal end-to-end fusion (21). In cancer, telomeres become dysfunctional due to the loss or alteration of telomere-binding proteins involved in telomere maintenance or to DNA damage caused by oxidative stress (22). The telomere-binding proteins TRF1 and TRF2 are crucial for the protection and maintenance of telomeres in mammalian cells $(8,23)$. TRF1 and TRF2 contain a Myb-like helix-turn-helix domain in the $\mathrm{C}$-terminus of the protein, and a conserved central domain that is responsible for the formation of homodimers (24). Previous studies have indicated that TRF1 and TRF2 are down-regulated in malignant tissues $(13,14,25-28)$. To our knowledge, the present study is the first to report not only that TRF1 and TRF2 are down-regulated in OCSCC, but also that their expression levels are correlated with the clinical characteristics of tumors. The correlation of TRF1 and TRF2 expression with clinical T stage may be explained at the cellular level by the roles of TRF1 and TRF2 in regulating the growth of cancer cells, while the correlation with $\mathrm{N}$ stage may reflect the participation of TRF1 and TRF2 in the control of metastasis. In contrast to our results, other studies have revealed that TRF1 and TRF2 are up-regulated 
in aggressive adenocarcinoma (29-32). This apparent disparity may be the result of differences in the tumors examined and in their microenvironments.

In the present study, decreased expression of TRF1 and TRF2 was detected in OCSCC patients based on Western blotting and immunohistochemistry. TRF1 and TRF2 were strongly expressed in the nucleus of adjacent non-cancerous tissues, and weakly expressed in human OCSCC specimens. Additionally, expression of TRF1 and TRF2 was correlated with 5-year overall survival and clinical prognosis. Notably, TRF1 expression was an independent prognostic indicator for OCSCC in this cohort. These results indicate that TRF1 and TRF2 may be critical regulators of disease progression in OCSCC, making them potential therapeutic targets. Future studies of the physiological targets of TRF1 and TRF2 and their potential roles in the pathogenesis of OCSCC may facilitate the development of novel therapeutic strategies.

\section{Acknowledgements}

This study was supported by Chang Gung Memorial Hospital, Taiwan (grant nos. CMRPG870411, CMRPG870412 and CMRPG860513).

\section{References}

1. Shah JP and Singh B: Why the lack of progress for oral cancer? Lancet Oncol 7: 356-357, 2006.

2. Chien CY, Su CY, Chuang HC, et al: Angiopoietin-1 and -2 expression in recurrent squamous cell carcinoma of the oral cavity. J Surg Oncol 97: 273-277, 2008.

3. Chen PH, Ko YC, Yang YH, et al: Important prognostic factors of long-term oropharyngeal carcinoma survivors in Taiwan. Oral Oncol 40: 847-855, 2004.

4. Matsuo JM, Patel SG, Singh B, et al: Clinical nodal stage is an independently significant predictor of distant failure in patients with squamous cell carcinoma of the larynx. Ann Surg 238: 412-421, 2003.

5. Chien CY, Su CY, Chuang HC, et al: Comprehensive study on the prognostic role of osteopontin expression in oral squamous cell carcinoma. Oral Oncol 45: 798-802, 2009.

6. Chen CH, Chien CY, Huang CC, et al: Expression of FLJ10540 is correlated with aggressiveness of oral cavity squamous cell carcinoma by stimulating cell migration and invasion through increased FOXM1 and MMP-2 activity. Oncogene 28: 2723-2737, 2009.

7. Vaziri H, Schachter F, Uchida I, et al: Loss of telomeric DNA during aging of normal and trisomy 21 human lymphocytes. Am J Hum Genet 52: 661-667, 1993.

8. Van Steensel B and de Lange T: Control of telomere length by the human telomeric protein TRF1. Nature 385: 740-743, 1997.

9. Smogorzewska A, van Steensel B, Bianchi A, et al: Control of human telomere length by TRF1 and TRF2. Mol Cell Biol 20: 1659-1668, 2000.

10. Sfeir A, Kosiyatrakul ST, Hockemeyer D, et al: Mammalian telomeres resemble fragile sites and require TRF1 for efficient replication. Cell 138: 90-103, 2009.

11. Van Steensel B, Smogorzewska A and de Lange T: TRF2 protects human telomeres from end-to-end fusions. Cell 92: 401-413, 1998.

12. Ancelin K, Brunori M, Bauwens S, et al: Targeting assay to study the cis functions of human telomeric proteins: evidence for inhibition of telomerase by TRF1 and for activation of telomere degradation by TRF2. Mol Cell Biol 22: 3474-3487, 2002.
13. Yamada K, Yagihashi A, Yamada M, et al: Decreased gene expression for telomeric-repeat binding factors and TIN2 in malignant hematopoietic cells. Anticancer Res 22: 1315-1320, 2002.

14. Yamada M, Tsuji N, Nakamura M, et al: Down-regulation of TRF1, TRF2 and TIN2 genes is important to maintain telomeric DNA for gastric cancers. Anticancer Res 22: 3303-3307, 2002.

15. Miyachi K, Fujita M, Tanaka N, Sasaki K and Sunagawa M: Correlation between telomerase activity and telomeric-repeat binding factors in gastric cancer. J Exp Clin Cancer Res 21: 269-275, 2002

16. Lin X, Gu J, Lu C, Spitz MR and Wu X: Expression of telomereassociated genes as prognostic markers for overall survival in patients with non-small cell lung cancer. Clin Cancer Res 12: 5720-5725, 2006.

17. Raynaud CM, Jang SJ, Nuciforo P, et al: Telomere shortening is correlated with the DNA damage response and telomeric protein down-regulation in colorectal preneoplastic lesions. Ann Oncol 19: 1875-1881, 2008.

18. Frias C, Garcia-Aranda C, De Juan C, et al: Telomere shortening is associated with poor prognosis and telomerase activity correlates with DNA repair impairment in non-small cell lung cancer. Lung Cancer 60: 416-425, 2008.

19. d'Adda di Fagagna F, Reaper PM, Clay-Farrace L, et al: A DNA damage checkpoint response in telomere-initiated senescence. Nature 426: 194-198, 2003.

20. Yajima T, Yagihashi A, Kameshima H, Kobayashi D, Hirata K and Watanabe N: Telomerase reverse transcriptase and telomericrepeat binding factor protein 1 as regulators of telomerase activity in pancreatic cancer cells. Br J Cancer 85: 752-757, 2001.

21. Salhab M, Jiang WG, Newbold RF and Mokbel K: The expression of gene transcripts of telomere-associated genes in human breast cancer: correlation with clinico-pathological parameters and clinical outcome. Breast Cancer Res Treat 109: 35-46, 2008.

22. Feldser DM, Hackett JA and Greider CW. Telomere dysfunction and the initiation of genome instability. Nat Rev Cancer 3: 623-627, 2003.

23. Chong L, van Steensel B, Broccoli D, et al: A human telomeric protein. Science 270: 1663-1667, 1995.

24. Broccoli D, Smogorzewska A, Chong L and de Lange T: Human telomeres contain two distinct Myb-related proteins, TRF1 and TRF2. Nat Genet 17: 231-235, 1997.

25. Saito K, Yagihashi A, Nasu S, Izawa Y, et al: Gene expression for suppressors of telomerase activity (telomeric-repeat binding factors) in breast cancer. Jpn J Cancer Res 93: 253-258, 2002.

26. Yamada K, Yajima T, Yagihashi A, et al: Role of human telomerase reverse transcriptase and telomeric-repeat binding factor proteins 1 and 2 in human hematopoietic cells. Jpn J Cancer Res 91: 1278-1284, 2000.

27. La Torre D, de Divitiis O, Conti A, et al: Expression of telomeric repeat binding factor-1 in astroglial brain tumors. Neurosurgery 56: 802-810, 2005

28. Kishi S, Wulf G, Nakamura M and Lu KP: Telomeric protein Pin2/TRF1 induces mitotic entry and apoptosis in cells with short telomeres and is down-regulated in human breast tumors. Oncogene 20: 1497-1508, 2001.

29. Oh BK, Kim YJ, Park C and Park YN: Up-regulation of telomerebinding proteins, TRF1, TRF2, and TIN2 is related to telomere shortening during human multistep hepatocarcinogenesis. Am J Pathol 166: 73-80, 2005.

30. Klapper W, Qian W, Schulte C and Parwaresch R: DNA damage transiently increases TRF2 mRNA expression and telomerase activity. Leukemia 17: 2007-2015, 2003.

31. Klapper W, Krams M, Qian W, Janssen D and Parwaresch R: Telomerase activity in B-cell non-Hodgkin lymphomas is regulated by hTERT transcription and correlated with telomerebinding protein expression but uncoupled from proliferation. $\mathrm{Br}$ J Cancer 89: 713-719, 2003.

32. Nakanishi K, Kawai T, Kumaki F, et al: Expression of mRNAs for telomeric repeat binding factor (TRF)-1 and TRF2 in atypical adenomatous hyperplasia and adenocarcinoma of the lung. Clin Cancer Res 9: 1105-1111, 2003. 